\title{
The association between daily exacerbation symptoms and physical activity in patients with chronic obstructive pulmonary disease
}

This article was published in the following Dove Press journal: International Journal of COPD

\author{
Sarah Crook' \\ Gilbert Büsching ${ }^{2}$ \\ Stephan Keusch ${ }^{3}$ \\ Stephan Wieser ${ }^{4}$ \\ Alexander Turk ${ }^{3,5}$ \\ Martin Frey ${ }^{2}$ \\ Milo A Puhan' \\ Anja Frei' \\ 'Department of Epidemiology, \\ Epidemiology, Biostatistics and \\ Prevention Institute, University \\ of Zurich, Zurich, Switzerland; \\ ${ }^{2}$ Pulmonary Rehabilitation, \\ Klinik Barmelweid, Barmelweid, \\ Switzerland; ${ }^{3}$ Pulmonology, Zürcher \\ RehaZentrum Wald, Wald, Switzerland; \\ ${ }^{4}$ Pulmonology, Stadtspital Waid, \\ Zurich, Switzerland; ${ }^{5}$ Department of \\ Internal Medicine, See-Spital Horgen, \\ Horgen, Switzerland
}

Correspondence: Anja Frei Department of Epidemiology, Epidemiology, Biostatistics and Prevention Institute, University of Zurich, Hirschengraben 84,

Zurich 800I, Switzerland

$\mathrm{Tel}+4$ I 446344360

Email anja.frei@uzh.ch
Background: Evidence from longitudinal studies on the impact of exacerbation symptoms on physical activity in chronic obstructive pulmonary disease (COPD) is lacking. The aim of this first exploratory study was to assess the association between exacerbation symptoms and physical activity, and to quantify the relative influence of specific symptoms.

Methods: We recruited COPD patients at high risk for exacerbations from 2 pulmonary rehabilitation clinics and 1 acute care clinic in Switzerland. For 3 months after discharge, patients completed a daily symptom diary on a smartphone application, the EXAcerbations of Chronic pulmonary disease Tool (EXACT), and wore a pedometer to measure daily steps. We used mixedeffects models to determine the association of daily steps with exacerbation symptoms.

Results: A total of 21 patients (Global Initiative for Chronic Obstructive Lung Disease grades 2-4) were enrolled for a mean of 94.4 days (standard deviation 4.2). The baseline median number of daily steps was 3,264.6 (interquartile range [IQR]: 1,851.3-4,784.1) and EXACT score was 37.0 (IQR: 30.9-41.4). A 12-point increase in EXACT score (indicating the start of an exacerbation) was statistically significantly associated with a decrease in daily steps of 653.3 (95\% CI 969.7-336.9). Chest symptoms (tightness, discomfort and congestion) were more strongly associated with change in steps than breathlessness, and cough and sputum ( $z$-value -4.5 vs -2.9 and -3.0 ).

Conclusion: This is the first study to show that, in a small cohort of COPD patients, increases in exacerbation symptoms were associated with a statistically and clinically significant reduction in daily physical activity. These results underscore the importance for symptom control and exacerbation prevention in COPD patients.

Keywords: longitudinal, management, step count, PRO, COPD, exacerbation, physical activity, symptoms

\section{Introduction}

Physical inactivity and exacerbations are both prominent features in chronic obstructive pulmonary disease (COPD) patients. They have been independently linked to outcomes including reduced health-related quality of life and increased morbidity and mortality. ${ }^{1-4}$ Physical activity is potentially modifiable and known determinants include age, sex and exercise capacity; ${ }^{5}$ however, there is still much to learn about factors influencing physical activity levels. ${ }^{3}$

The relationship between exacerbations of COPD and physical activity is complex and currently not well understood. Low activity levels are a strong predictor of future severe exacerbations $;{ }^{6-10}$ however, physical activity is also known to be reduced during exacerbations. ${ }^{11-13}$ Exacerbations are characterized by a worsening of the 
patient's respiratory symptoms beyond the normal day-today variability; ${ }^{14}$ however, there are a few studies published on exacerbation symptoms in relation to physical activity. Exacerbation symptoms such as increased cough, sputum production, dyspnea and chest congestion could plausibly limit patients' ability and/or willingness to be active. A recent systematic review found little evidence on the association between symptoms of COPD and objectively measured physical activity. ${ }^{15}$ One cross-sectional study reported statistically significant relationships between symptoms at different times of the day and subjectively assigned physical activity levels at baseline, ${ }^{16}$ and two studies found that reporting a sore throat was associated with a decline in physical activity during an exacerbation. ${ }^{12,17}$

However, to our knowledge the impact of specific symptoms on changes in physical activity has not been explicitly assessed in longitudinal studies beyond limited periods of time (ie, during hospitalization). As exacerbation symptoms are known to naturally vary daily both inside and outside of exacerbation periods, ${ }^{18}$ it is important to capture the relationship between symptoms and physical activity longitudinally across the natural course of COPD. Therefore, we aimed to investigate the association between exacerbation symptoms and physical activity in COPD patients, and to quantify the relative influence of specific respiratory symptoms.

\section{Methods}

\section{Study design and population}

We recruited patients from 2 pulmonary rehabilitation clinics (Klinik Barmelweid and Zürcher RehaZentrum Wald) and 1 acute care clinic (Stadtspital Waid) in Switzerland (ClinicalTrials.gov: NCT02441725). In Switzerland, patients follow an inpatient rehabilitation program typically just after suffering from an exacerbation, and these patients usually have moderate to very severe COPD and multiple comorbidities. Thus, the patients included in this study were at high risk for additional exacerbations. We recruited patients from these settings as they were more likely to experience exacerbations during the study period. Patients from the pulmonary rehabilitation clinics already participated in the Validation of the 1-minute Sit-to-Stand Test in Patients with COPD (STAND-UP) study, ${ }^{19}$ with assessments during rehabilitation. They then decided whether to participate in the present study, the extended part of the STAND-UP study with additional assessments for 3 months after discharge. We included male and female patients $\geq 40$ years of age with a COPD diagnosis according to Global Initiative for Chronic Obstructive Lung Disease criteria (post-bronchodilator forced expiratory volume in $1 \mathrm{~s}\left[\mathrm{FEV}_{1}\right] /$ forced vital capacity $<70 \%$ ). Patients were not included if the enrolling physician deemed them to have cognitive reading impairment and/or difficulties managing a smartphone or step counter that would limit their ability to complete the study assessments. Other exclusion criteria were lower limb joint surgery in the preceding 3 months, predominant neurological or musculoskeletal limitation to walking and inability to do five repetitions in the 1-minute sit-to-stand test.

This study was approved by all local ethics committees (Kantonale Ethikkommission Zürich [2014-0614] and Ethikkommission Nordwest-und ZentralSchweiz [2015095]). All patients gave their written and oral informed consent to participate.

\section{Daily assessments}

Every evening before going to bed, participants completed the EXAcerbations of Chronic pulmonary disease Tool (EXACT) on a smartphone application, which is a validated 14-item symptom diary. ${ }^{20}$ The EXACT diary was developed and validated in a multistep process by the EXACT-PRO Initiative $^{\circledR}$. EXACT aims to provide a direct measure of exacerbation symptoms to evaluate the frequency, severity and duration of exacerbations in COPD patients. It asks about symptoms including cough, sputum production, dyspnea, chest symptoms (tightness, discomfort and congestion), tiredness, weakness, sleep disturbance and psychological state, and participants answer based on the symptoms experienced that day. The total EXACT score reflects the entirety of the symptoms on a scale of 0-100, with higher scores indicating more severe symptoms. ${ }^{20,21}$ An increase from baseline of 9 (for 3 days) or 12 points (for 2 days) is used to identify the start of an EXACT event (symptom-defined exacerbation). The Evaluating Respiratory Symptoms in COPD (E-RS) tool is a validated sub-scale of the EXACT, which reflects the specific respiratory symptoms experienced in three domains (breathlessness [scale 0-17, 5 items], cough and sputum $\left[0-11,3\right.$ items], and chest symptoms [0-12, 3 items]). ${ }^{22,23}$ Participants were given a study smartphone to use if they did not have/wish to use their own.

Participants wore a pedometer (Fitbug Air; Fitbug, London, UK) on the hip for 3 months, at all times, except when sleeping or showering/swimming. They were asked to transfer the steps data each evening to the Fitbug smartphone application; if 1 or more days were missed, steps data were stored on the pedometer for 14 days. We trained participants to use the smartphone, the EXACT application, and to transfer the steps data. To maximize compliance, data transfers 
were monitored by study staff and participants were contacted if $\geq 3$ days were missed.

\section{Event-based exacerbations and adverse events}

Exacerbations and adverse events were assessed with monthly telephone calls that were made to the patient by an experienced study nurse. The event-based definition required an increase in symptoms and an increase in dosage of or new prescription of systemic corticosteroids and/or antibiotics.

\section{Statistical analyses}

Data were expressed as mean \pm standard deviation (SD) or median (interquartile range [IQR]), depending on the data distribution. We used linear mixed-effects models with daily step count as the outcome, with a random intercept by subjects and a random slope to account for the repeated measurements of exacerbation symptoms and step count within patients. To investigate exacerbation symptoms and physical activity, we fitted a model with the daily total EXACT score as the exposure variable and the random slope (model 1). We fitted three further models, one for each E-RS domain as the exposure variable and random slope, to investigate the relative influence of different respiratory symptoms (models 2-4). All models were adjusted for relevant covariates that we identified from clinical experience and previous research ${ }^{3,5}$ (age, sex, $\mathrm{FEV}_{1}[\mathrm{~L}]$, body mass index, number of exacerbations in the previous year, number of comorbidities, smoking status and functional exercise capacity [1-minute sit-to-stand test repetitions]). All models were estimated with an unstructured covariance structure to allow for correlation between the two random effects. Due to heteroskedasticity of the residuals, we used a robust variance estimator to estimate the standard errors. For model 1, we estimated the change in daily steps in relation to a change in EXACT score of 12 points and 9 points, and for all models we calculated the $z$-value and Bayesian Information Criterion (BIC). Analyses were conducted using Stata (version 14.1; StataCorp, College Station, TX, USA).

\section{Results}

\section{Population characteristics}

In total, 22 patients from 2 pulmonary rehabilitation clinics $(n=19)$ and 1 acute care hospital $(n=3)$ with moderate to very severe COPD were included in the study. One patient withdrew from the study after baseline assessment due to a lack of motivation. Patients were followed up for a mean of 94.4 (SD 4.2) days. Table 1 summarizes the baseline patient characteristics.
Table I Patient characteristics at discharge from pulmonary rehabilitation or acute care

\begin{tabular}{|c|c|}
\hline Characteristics & Patients $(\mathrm{N}=\mathbf{2} \mathrm{I})$ \\
\hline Sex, male, n (\%) & $14(66.7)$ \\
\hline Age (years) & $63.0(9.9)$ \\
\hline $\mathrm{FEV}_{1}(\mathrm{~L})$ & $\mathrm{I} .2(0.4)$ \\
\hline $\mathrm{FEV}_{1} \%$ predicted & $40.8(14.3)$ \\
\hline BMI $\left(\mathrm{kgm}^{-2}\right)$ & $23.9(6.1)$ \\
\hline \multicolumn{2}{|l|}{ Smoking status, n (\%) } \\
\hline Never-smoker & I (4.8) \\
\hline Former smoker & $15(7 \mid .4)$ \\
\hline Current smoker & $5(23.8)$ \\
\hline \multicolumn{2}{|l|}{ GOLD grade, n (\%) } \\
\hline II & $5(23.8)$ \\
\hline III & $8(38.1)$ \\
\hline IV & $8(38.1)$ \\
\hline Exacerbations in previous year & $2.3(1.9)$ \\
\hline Number of comorbidities per patient & $3.4(1.9)$ \\
\hline \multicolumn{2}{|l|}{ Comorbidity type, $n$} \\
\hline Cardiovascular disease group & 25 \\
\hline Cerebrovascular disease group & 1 \\
\hline Metabolic disease group & 1 \\
\hline Musculoskeletal disease group & 5 \\
\hline Psychiatric disease group & 6 \\
\hline Other diseases & 31 \\
\hline I-min STS test repetitions & $22.4(8.0)$ \\
\hline
\end{tabular}

Note: Data are presented as mean (SD), unless otherwise stated.

Abbreviations: $\mathrm{BMI}$, body mass index; $\mathrm{FEV}_{1}$, forced expiratory volume in I s; GOLD, Global Initiative for Chronic Obstructive Lung Disease; I-min STS, I-minute sit-to-stand.

\section{Baseline outcomes and adverse events}

Participants had a mean of 85.3 (SD 17.3) days of step counts and 77.7 (SD 20.1) days of EXACT scores. Eleven eventbased exacerbations (9 home-based and 2 hospital-based) were experienced by 9 patients, and 10 adverse events were experienced by 6 patients (all unrelated to the study assessments, non-serious and did not require hospitalization). The median (IQR) baseline daily step count was 3,264.6 (1,851.34,784.1) and EXACT score was 37.0 (30.9-41.4), calculated as the median daily value of a stable period of 7 days (first week after discharge for pulmonary rehabilitation patients and second week after discharge for acute care patients). The EXACT scoring algorithm used to determine a symptomdefined exacerbation did not detect $>50 \%$ of the event-based exacerbations in our data. These results reflect those found previously in an independent validation of the EXACT scoring algorithm. ${ }^{24}$ Therefore, we did not consider symptom-defined exacerbations in the interpretation of our data.

\section{Exacerbation symptoms}

Table 2 shows the adjusted coefficient for the association between daily EXACT score and daily step count, along 
Table 2 Multivariable analysis assessing the association between daily EXACT score and daily physical activity, adjusted for relevant confounders (model I)

\begin{tabular}{|c|c|c|c|c|}
\hline Variable & Effect & $95 \% \mathrm{Cl}$ & z-value & $P$-value \\
\hline \multicolumn{5}{|l|}{ Daily EXACT score ${ }^{a}$} \\
\hline I point increase & -54.4 & -80.8 to -28.1 & -4.1 & $<0.001$ \\
\hline 9 point increase & -490.0 & -727.3 to -252.7 & -4.1 & $<0.001$ \\
\hline 12 point increase & -653.3 & -969.7 to -336.9 & -4.1 & $<0.001$ \\
\hline Age (years) & 1.0 & -36.0 to 37.9 & 0.1 & 0.96 \\
\hline \multicolumn{5}{|l|}{ Sex (ref: male) } \\
\hline Female & 74.3 & -952.3 to $1,101.0$ & 0.1 & 0.89 \\
\hline $\mathrm{FEV}_{1}(\mathrm{~L})$ & 820.7 & 145.5 to $\mid, 496.0$ & 2.4 & 0.02 \\
\hline BMI $\left(\mathrm{kgm}^{-2}\right)$ & -34.8 & -112.8 to 43.1 & -0.9 & 0.38 \\
\hline I-min STS test repetitions & 49.6 & -2.5 to 101.7 & 1.9 & 0.06 \\
\hline Number of comorbidities per patient & -471.7 & $-7 \mid 7.1$ to -226.2 & -3.8 & $<0.001$ \\
\hline \multicolumn{5}{|l|}{ Smoking status (ref: current-smoker) } \\
\hline Former smoker & $1,070.0$ & 73.7 to $2,066.3$ & 2.1 & 0.04 \\
\hline Non-smoker & $3,968.5$ & $2,988.1$ to $4,948.8$ & 7.9 & $<0.001$ \\
\hline Number of exacerbations in the previous year & -8.3 & -150.4 to 133.7 & -0.1 & 0.91 \\
\hline
\end{tabular}

Notes: aScore range: 0-100 points. Bayesian Information Criterion =25,709.

Abbreviations: BMI, body mass index; EXACT, EXAcerbations of Chronic pulmonary disease Tool; FEV , forced expiratory volume in I s; I-min STS, I-minute sit-to-stand; ref, reference.

with the coefficients for each covariate. A 9-point increase in daily EXACT score was associated with a significant decrease of $-490.0(95 \% \mathrm{CI}-727.3$ to -252.7$)$ in the number of daily steps. A 12-point increase in daily EXACT score was associated with a decrease of -653.3 (95\% CI -969.7 to -336.9 ) in the number of daily steps. In model 1 , the adjusted intraclass correlation coefficient was 0.25 . This means that most of the variation in daily steps (75\%) was within individuals and only $25 \%$ was between individuals. The fitted regression lines from model 1 show the predicted association between daily exacerbation symptoms and daily steps for each patient (Figure 1). There is variation between

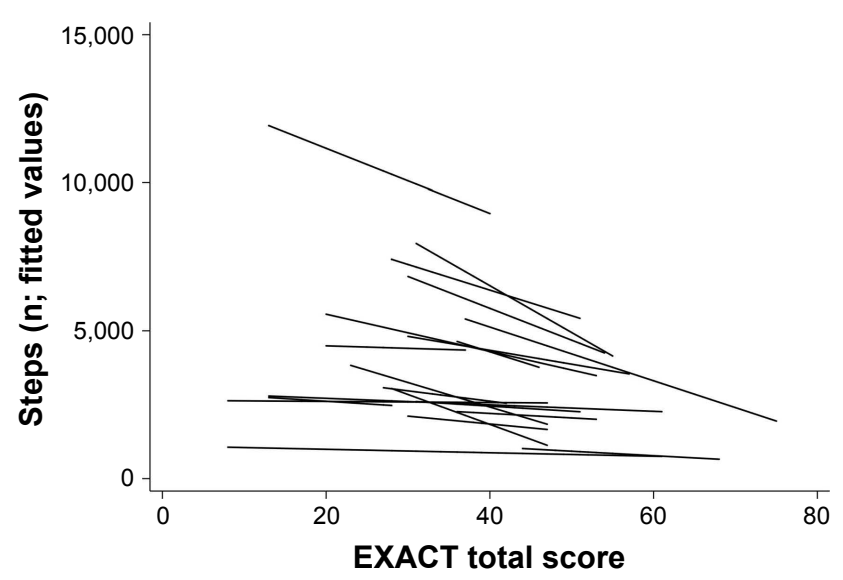

Figure I Fitted regression lines for individual patients for the association between daily step count and daily EXACT score from model I. The figure shows the predicted number of daily steps for the given EXACT score. There appears to be a weaker association (slope more horizontal) at higher numbers of steps, and more between-patient variation at lower EXACT scores (fewer symptoms).

Abbreviation: EXACT, EXAcerbations of Chronic pulmonary disease Tool. patients in the estimated intercept and slope of the association. There also appears to be a weaker association (slope more horizontal) at higher numbers of steps, and more between-patient variation at lower EXACT scores (fewer symptoms).

All three domains of the E-RS were independently significantly associated with the number of daily steps in adjusted models (Table 3). The standardized regression coefficients show that the effect of chest symptoms on the number of daily steps was stronger than the effects of breathlessness, and cough and sputum (adjusted $z$-value -4.5 vs -2.9 and -3.0 , respectively). The BIC was lowest for the model with chest symptoms, indicating there is a better fit of the data (lower BIC) than the models with the breathlessness and cough and sputum domains (breathlessness +17 and cough and sputum +12 , compared to chest symptoms).

Individual patient courses of daily steps and daily EXACT scores over the 3-month study period are shown in Figure 2. Step count and EXACT score are presented as 5-day moving averages to facilitate the visualization of trends.

\section{Discussion}

In this novel study, we found that an increase in exacerbation symptoms was strongly associated with a reduction in physical activity. The strength of this association was found to differ between patients. Chest symptoms (tightness, discomfort and congestion) were more strongly associated with changes in physical activity than other respiratory symptoms.

To our knowledge, this is the first study to investigate the impact of exacerbation symptoms on changes in physical 
Table 3 Adjusted $^{\mathrm{a}}$ associations of each respiratory symptom domain with daily physical activity, estimated from individual models (models 2-4)

\begin{tabular}{|c|c|c|c|c|c|}
\hline E-RS symptom domain score & Effect & $95 \% \mathrm{Cl}$ & z-value & $P$-value & BIC \\
\hline Breathlessness $^{\mathrm{b}}$ (model 2$)$ & -153.4 & -257.8 to -49.0 & -2.9 & 0.004 & 25,748 \\
\hline Cough and sputum (model 3) & -127.8 & -212.7 to -42.9 & -3.0 & 0.003 & 25,743 \\
\hline Chest symptoms ${ }^{\mathrm{b}, \mathrm{c}}($ model 4$)$ & -187.7 & -268.8 to -106.7 & -4.5 & $<0.001$ & 25,731 \\
\hline
\end{tabular}

Notes: aAll 3 models were adjusted for the same confounders as in model I; ball effects are per I-point increase. Breathlessness score range: 0 -I7 points, cough and sputum score range: 0-II points, chest symptoms score range: 0-12 points; 'chest symptoms include chest tightness, discomfort and congestion.

Abbreviations: BIC, Bayesian Information Criterion; E-RS, Evaluating Respiratory Symptoms in chronic obstructive pulmonary disease.

activity on a day-to-day basis. Patients were less likely to be active when they were experiencing exacerbation symptoms, resulting in a strong and statistically significant reduction in the number of steps walked. A clinically important increase in symptoms of exacerbation (an increase in total EXACT score of 12 points) ${ }^{20}$ was associated with a reduction of 653 steps on that day. This reduction can be considered as clinically important as it exceeds the lower threshold of the recently estimated minimum important difference for daily step count (600-1,100 steps). ${ }^{25}$
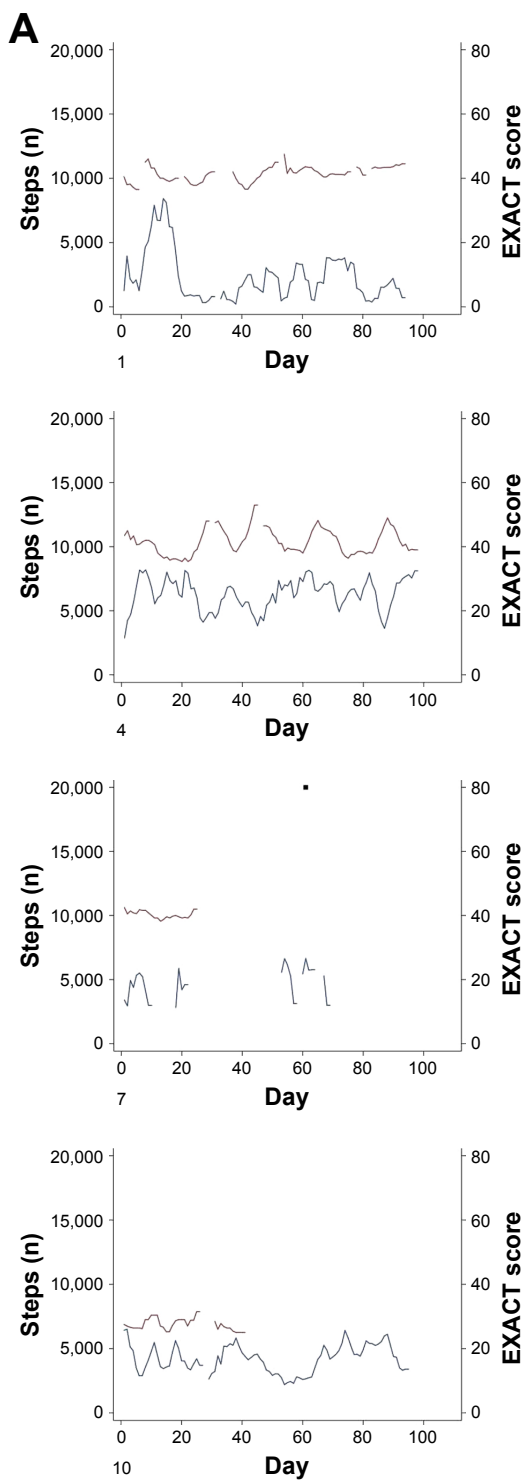
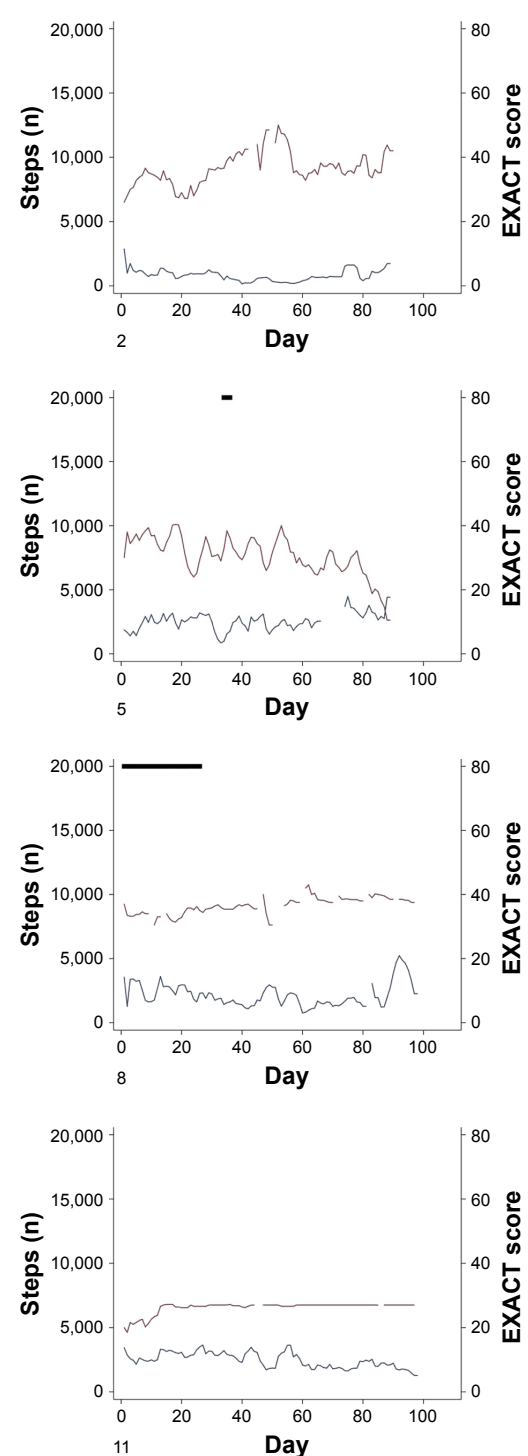
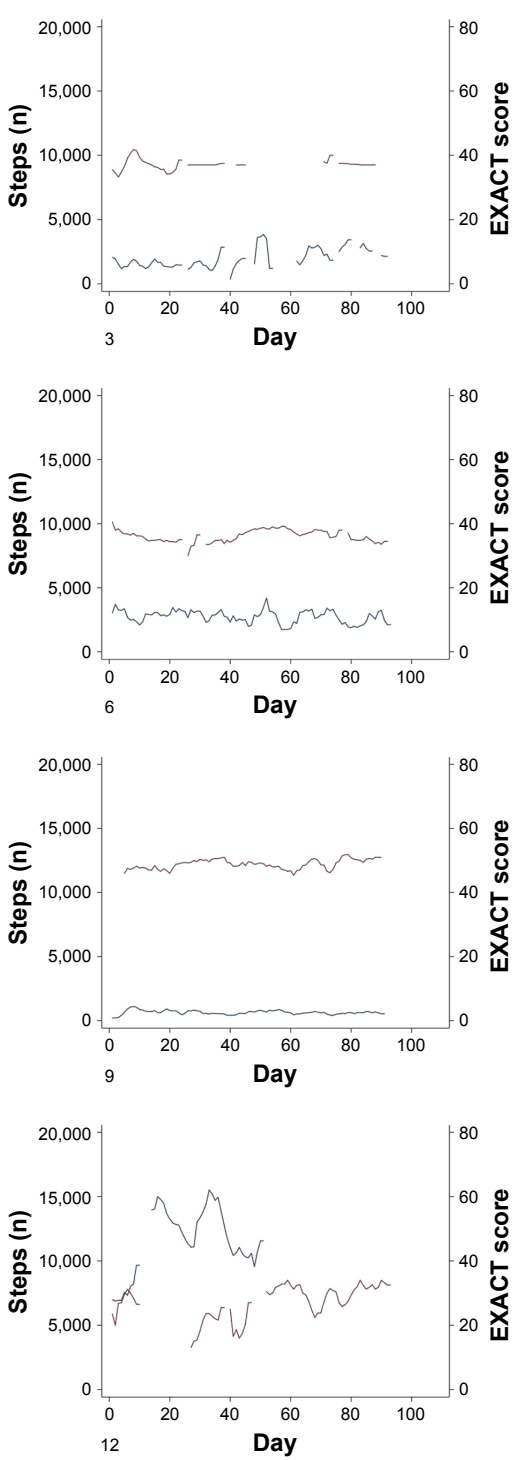

EXACT score $\longrightarrow$ Steps $(n) \bullet$ Exacerbation start - Adverse event

Figure 2 (Continued) 

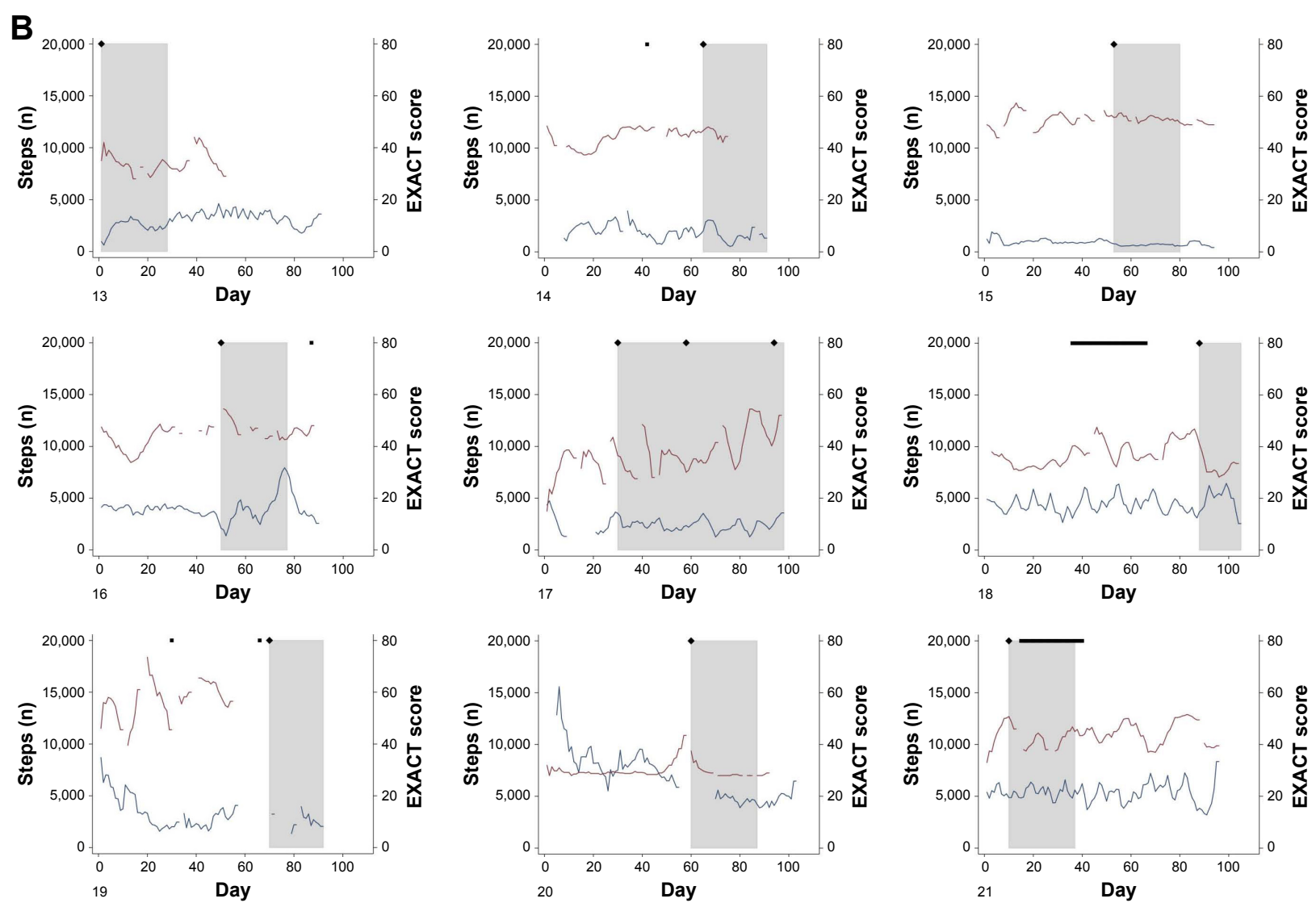

EXACT score $\longrightarrow$ Steps $(n) \bullet$ Exacerbation start $\quad$ Adverse event $\quad$ Exacerbation recovery

Figure 2 Individual courses of daily step count and daily total EXACT score over the 3-month study period for patients who did not have an exacerbation (A) and patients who did have an exacerbation (B). Diamonds indicate the start date of clinically defined event-based exacerbations, gray boxes indicate the 28-day recovery period after the start of an exacerbation and black squares indicate adverse events (unrelated to COPD exacerbations). Patients I6 ( 14 days) and I9 (I day) were admitted to hospital due to their exacerbations; all other exacerbations were home-based. Adverse events were constipation (patient 5), numbness in feet (patient 7), purulent infection on the arm (8), stomach pain and diarrhea (14), electric cardioversion (16), severe sunburn to chest (18), tooth removal and armpit abscess (19) and crushed ribs (2I). No adverse events required hospitalization.

Notes: Numbers in the lower left-hand corner represent an individual patient (in random order).

Abbreviations: COPD, chronic obstructive pulmonary disease; EXACT, EXAcerbations of Chronic pulmonary disease Tool.

Figures 1 and 2 indicate that the strength of the association between exacerbation symptoms and physical activity differs among patients. Reasons for these inter-patient differences are currently unclear; however, there may be some influence from the average number of daily steps or overall variability in the number of daily steps, as suggested by the differences between estimated intercepts and slopes (Figure 1). It could be that patients with on average higher steps or with more variation in steps are influenced by exacerbation symptoms in a stronger manner, as their activity levels are able to reduce more than those who have an already low level, or it could be that another factor, such as severity of COPD, is driving the between-patient differences. One previous study found that patients with more daily and weekly variability in symptoms were more affected in their daily activities. ${ }^{18}$ However, this would need to be investigated in a larger study of COPD patients. The association between EXACT score and number of steps is evident in some patients but is not always obvious (Figure 2). Patient 4 is an example of a seemingly consistent negative correlation, whereas patient 2 appears to show little correlation. There is marked variability in exacerbation symptoms even in periods with no event-based exacerbation, which corresponds with a previous study that found daily and weekly variability in exacerbation symptoms in non-exacerbation periods. ${ }^{18}$

Previous research has shown that breathlessness and cough and sputum production are more commonly reported by COPD patients than chest discomfort and tightness. ${ }^{18,26}$ More patients also perceived increasing cough, breathlessness and sputum to have a high impact on wellbeing than chest pain ( $42 \%, 37 \%$ and $35 \%$ vs $20 \%$ of respondents). ${ }^{26}$ However, in our patients we found that an increase in chest symptoms had a more substantial impact on physical activity than breathlessness or cough and sputum. We can only speculate about the specific underlying cause of these 
symptoms, since a high number of patients in our study had cardiovascular comorbidities. Although EXACT was specifically developed in COPD patients to assess symptoms of respiratory exacerbations, it cannot differentiate the origins of the symptoms reported. Our results also differ from two studies where the only symptoms found to be associated with decline in physical activity were sore throat alone or sore throat and dyspnea or cough combined, although chest tightness, discomfort and congestion were not assessed and associations were only considered at exacerbation onset or during recovery. ${ }^{12,17}$ When considering symptoms in relation to interventions for improving physical activity levels in COPD patients, it may be important to target the reduction of chest symptoms, regardless of the underlying mechanism.

We have provided novel individual patient data on the association between daily symptoms and daily physical activity during a typical 3-month period. Previous research has focused on physical activity during or before exacerbations, or physical activity as a predictor for future exacerbations. ${ }^{6-9,11}$ Our study followed patients for 3 months, and therefore consists of periods of stability, exacerbation and recovery from exacerbation. Previous studies on the effect of exacerbations on physical activity have limited or categorized their analyses of physical activity to specific patient states (eg, only in stability or recovery vs exacerbation). ${ }^{12,13,17,18,27}$ A strength of our study is that we considered the overall association using longitudinal analyses, including different patient states and therefore avoiding the loss of valuable information. A potential additional hypothesis that should be explored in a larger study using longitudinal data and methods is whether the observed association differs during periods of exacerbation and stability.

One limitation of our study is that there was some missing data for daily steps and EXACT scores, which is difficult to avoid in longitudinal studies with repeated assessments. In some instances, the data were missing due to technical problems with the smartphone and/or application (missing completely at random). However, the reason for missingness was often not clear. While the mixed-effects model accounts for missing repeated-measures data, it should be considered that there may be important information missing when the reason for missing values was related to the outcome (ie, patient not completing the EXACT questionnaire during an exacerbation). A second limitation is the small sample size, which limits our ability to investigate the association further. This is partly explained by the substantial commitment patients need to have to report symptoms daily and wear a pedometer for 3 months. Despite this, we used longitudinal methods that enabled us to make use of the large amount of data collected over the study period. In total, model 1 utilized around 1,500 observations. Therefore, we consider our results to be valid in this sample, and our results may be generalizable to other patients with moderate to very severe COPD following an exacerbation. From visual inspection of individual patient data, we generated further hypotheses concerning the relationship between exacerbation symptoms and physical activity, although the small number of clusters limited our ability to explore these. Our use of pedometers to measure physical activity rather than accelerometers could be considered as a limitation since pedometers may underestimate steps in moderate to very severe COPD patients ${ }^{28,29}$ and we do not have additional information on the intensity or duration of activity. However, as the patients in our study all had moderate to very severe COPD, it is likely that bias from underestimation of steps is similar across patients. Therefore, we decided to use the Fitbug Air as a simple tool for assessing physical activity, which has previously been used in studies of COPD patients, ${ }^{30}$ as it is more valid than self-reported questionnaires, has less burden for patients and can record activity over a longer period than accelerometers. ${ }^{31}$

\section{Conclusion}

An increase in daily exacerbation symptoms was statistically and clinically significantly associated with increasing physical inactivity in our population of COPD patients. Respiratory chest symptoms may be more important in determining physical inactivity levels than other respiratory symptoms. These novel results give us additional insight into the behavior of COPD patients in relation to changes in physical activity and emphasize the need for symptom control and prevention of exacerbations.

\section{Acknowledgments}

The authors would like to thank the study nurses Ursula Schafroth (University of Zurich, Zurich, Switzerland) and Luise Wiedemann (Zürcher RehaZentrum Wald, Wald, Switzerland) for their work during the conduct of the study. We also thank Verein Lunge Zürich for providing the funding for the STAND-UP study. The funding body had no role in the design of the study, the collection and analysis of the data or the preparation of the manuscript. We would also like to thank Swisscom AG for donating the mobile phone SIM cards that were used for data collection during the study and Professor Doctor Ulf Blanke (Wearable Computing Laboratory, ETH Zurich, Swiss Federal Institute of Technology, Zurich, Switzerland) for developing the smartphone application and software for collecting the EXACT and steps data. 


\section{Disclosure}

The authors report no conflicts of interest in this work.

\section{References}

1. Langsetmo L, Platt RW, Ernst P, Bourbeau J. Underreporting exacerbation of chronic obstructive pulmonary disease in a longitudinal cohort. Am J Respir Crit Care Med. 2008;177(4):396-401.

2. Anzueto A, Leimer I, Kesten S. Impact of frequency of COPD exacerbations on pulmonary function, health status and clinical outcomes. Int J Chron Obstruct Pulmon Dis. 2009;4:245-251.

3. Gimeno-Santos E, Frei A, Steurer-Stey C, et al; PROactive consortium. Determinants and outcomes of physical activity in patients with COPD: a systematic review. Thorax. 2014;69(8):731-739.

4. Van Remoortel H, Hornikx M, Langer D, et al. Risk factors and comorbidities in the preclinical stages of chronic obstructive pulmonary disease. Am J Respir Crit Care Med. 2014;189(1):30-38.

5. Yu T, Frei A, Ter Riet G, Puhan MA. Determinants of physical activity in patients with chronic obstructive pulmonary disease: a 5-year prospective follow-up study. Respiration. 2016;92(2):72-79.

6. Garcia-Aymerich J, Farrero E, Félez MA, Izquierdo J, Marrades RM, Antó JM; Regular physical activity reduces hospital admission and mortality in chronic obstructive pulmonary disease: a population based cohort study. Risk factors of readmission to hospital for a COPD exacerbation: a prospective study. Thorax. 2003;58(2):100-105.

7. Garcia-Aymerich J, Lange P, Benet M, Schnohr P, Antó JM. Regular physical activity reduces hospital admission and mortality in chronic obstructive pulmonary disease: a population based cohort study. Thorax. 2006;61(9):772-778.

8. Moy ML, Teylan M, Weston NA, Gagnon DR, Garshick E. Daily step count predicts acute exacerbations in a US cohort with COPD. PLoS One. 2013;8(4):e60400.

9. Garcia-Rio F, Rojo B, Casitas R, et al. Prognostic value of the objective measurement of daily physical activity in patients with COPD. Chest. 2012;142(2):338-346.

10. Nguyen HQ, Rondinelli J, Harrington A, et al. Functional status at discharge and 30-day readmission risk in COPD. Respir Med. 2015; 109(2):238-246

11. Pitta F, Troosters T, Probst VS, Spruit MA, Decramer M, Gosselink R. Physical activity and hospitalization for exacerbation of COPD. Chest. 2006;129(3):536-544.

12. Donaldson GC, Wilkinson TM, Hurst JR, Perera WR, Wedzicha JA. Exacerbations and time spent outdoors in chronic obstructive pulmonary disease. Am J Respir Crit Care Med. 2005;171(5):446-452.

13. Ehsan M, Khan R, Wakefield D, et al. A longitudinal study evaluating the effect of exacerbations on physical activity in patients with chronic obstructive pulmonary disease. Ann Am Thorac Soc. 2013;10(6):559-564.

14. Vestbo J, Hurd SS, Agustí AG, et al. Global strategy for the diagnosis, management, and prevention of chronic obstructive pulmonary disease: GOLD executive summary. Am J Respir Crit Care Med. 2013;187(4):347-365.

15. Miravitlles M, Ribera A. Understanding the impact of symptoms on the burden of COPD. Respir Res. 2017;18(1):67.

16. Miravitlles M, Worth H, Soler Cataluña JJ, et al. Observational study to characterise 24-hour COPD symptoms and their relationship with patient-reported outcomes: results from the ASSESS study. Respir Res. 2014;15(1):122.

International Journal of COPD

\section{Publish your work in this journal}

The International Journal of COPD is an international, peer-reviewed journal of therapeutics and pharmacology focusing on concise rapid reporting of clinical studies and reviews in COPD. Special focus is given to the pathophysiological processes underlying the disease, intervention programs, patient focused education, and self management protocols.
17. Alahmari AD, Patel AR, Kowlessar BS, et al. Daily activity during stability and exacerbation of chronic obstructive pulmonary disease. BMC Pulm Med. 2014;14(1):98.

18. Kessler R, Partridge MR, Miravitlles M, et al. Symptom variability in patients with severe COPD: a pan-European cross-sectional study. Eur Respir J. 2011;37(2):264-272.

19. Crook S, Büsching G, Schultz K, et al. A multicentre validation of the 1-min sit-to-stand test in patients with COPD. Eur Respir J. 2017;49(3). pii: 1601871.

20. Leidy NK, Wilcox TK, Jones PW, Roberts L, Powers JH, Sethi S; EXACTPRO Study Group. Standardizing measurement of chronic obstructive pulmonary disease exacerbations. Reliability and validity of a patientreported diary. Am J Respir Crit Care Med. 2011;183(3):323-329.

21. Leidy NK, Wilcox TK, Jones PW, et al; EXACT-PRO Study Group. Development of the EXAcerbations of Chronic Obstructive Pulmonary Disease Tool (EXACT): a patient-reported outcome (PRO) measure. Value Health. 2010;13(8):965-975.

22. Leidy NK, Murray LT, Monz BU, et al. Measuring respiratory symptoms of COPD: performance of the EXACT-Respiratory Symptoms Tool (E-RS) in three clinical trials. Respir Res. 2014;15(1):124.

23. Leidy NK, Sexton CC, Jones PW, et al. Measuring respiratory symptoms in clinical trials of COPD: reliability and validity of a daily diary. Thorax. 2014;69(5):443-449.

24. Mackay AJ, Donaldson GC, Patel AR, Singh R, Kowlessar B, Wedzicha JA. Detection and severity grading of COPD exacerbations using the exacerbations of chronic pulmonary disease tool (EXACT). Eur Respir J. 2014;43(3):735-744.

25. Demeyer H, Burtin C, Hornikx M, et al. The minimal important difference in physical activity in patients with COPD. PLoS One. 2016; 11(4): $\mathrm{e} 0154587$.

26. Miravitlles M, Anzueto A, Legnani D, Forstmeier L, Fargel M. Patient's perception of exacerbations of COPD - the PERCEIVE study. Respir Med. 2007;101(3):453-460.

27. Alahmari AD, Kowlessar BS, Patel AR, et al. Physical activity and exercise capacity in patients with moderate COPD exacerbations. Eur Respir J. 2016;48(2):340-349.

28. Furlanetto KC, Bisca GW, Oldemberg N, et al. Step counting and energy expenditure estimation in patients with chronic obstructive pulmonary disease and healthy elderly: accuracy of 2 motion sensors. Arch Phys Med Rehabil. 2010;91(2):261-267.

29. Turner LJ, Houchen L, Williams J, Singh SJ. Reliability of pedometers to measure step counts in patients with chronic respiratory disease. J Cardiopulm Rehabil Prev. 2012;32(5):284-291.

30. Demeyer H, Louvaris Z, Frei A, et al; Mr Papp PROactive study group and the PROactive consortium. Physical activity is increased by a 12-week semiautomated telecoaching programme in patients with COPD: a multicentre randomised controlled trial. Thorax. 2017;72(5):415-423.

31. Watz H, Pitta F, Rochester CL, et al. An official European Respiratory Society statement on physical activity in COPD. Eur Respir J. 2014;44(6): 1521-1537.

This journal is indexed on PubMed Central, MedLine and CAS. The manuscript management system is completely online and includes a very quick and fair peer-review system, which is all easy to use. Visit http://www.dovepress.com/testimonials.php to read real quotes from published authors. 